\title{
Análise da incidência de óbitos fetais entre municípios da nona regional de saúde do Paraná
}

\section{Analysis of the incidence of fetal deaths between cities the ninth regional health of Paraná}

\author{
Rosane Meire Munhak Silva ${ }^{1}$, Bruna Ribeiro Mazotti ${ }^{2}$, Adriana Zilly ${ }^{3}$, Helder \\ Ferreira $^{4}$, Sebastião Caldeira ${ }^{5}$
}

\begin{abstract}
Resumo
O objetivo do estudo foi analisar os óbitos fetais entre municípios da nona regional de saúde do Paraná. Pesquisa descritiva com abordagem quantitativa. A coleta aconteceu no primeiro semestre de 2015 , no Sistema de Informação de Nascidos Vivos, no Sistema de Informação sobre Mortalidade e nos relatórios do Comitê de Mortalidade Materno e Infantil da nona Regional de Saúde do Paraná. Cinco municípios tiveram aumento nos óbitos fetais entre 2010 e 2013, sendo que, Itaipulândia apresentou os piores índices e Santa Terezinha de Itaipu um aumento de cinco vezes. Entre os óbitos fetais, destacaram-se fetos do sexo masculino, com peso entre 500 e 1499 gramas e idade gestacional de 28 a 36 semanas. Quanto à idade materna, predominou a faixa etária de 20 a 39 anos. Em 2010, a maior parte dos óbitos fetais acorreu com mães de baixa escolaridade, já em 2013, não se observou esta relação. Para a maioria, o tipo de parto foi vaginal, mas ainda se observa que o parto cirúrgico aconteceu em muitos casos de óbito fetal. A construção de estudos sobre a saúde fetal com diferentes enfoques de abordagem torna-se relevante, pois estes têm sido pouco explorados, apesar de sua incontestável importância em termos de saúde pública como indicador da qualidade da assistência pré-natal e ao parto.
\end{abstract}

Palavras chave: Morte fetal. Parto. Fatores de risco.

\begin{abstract}
The aim of this study was to analyze stillbirth between municipalities of the ninth regional Health of Paraná. This was a descriptive research with quantitative approach. The data collection occurred in 2015, from the Brazilian National Information System on Live Births, in Mortality information system and in reports of the Maternal and Infant Mortality Committee of the ninth Regional Health of Paraná. Five municipalities had an increase in stillbirths between 2010 and 2013. Itaipulândia showed the highest incidence and Santa Terezinha de Itaipu showed a fivefold increase of stillbirths. Most stillbirths were male fetuses, weighing 500-1499 grams and had gestational age between 28-36 weeks. As for maternal age, stillbirths were the most common between 20-39 years. In 2010, most stillbirths occurred with least educated mothers, while in 2013 there was no significant difference between educational levels. Although the majority of deliveries were vaginal, surgical birth happened in many cases. Different
\end{abstract}

\footnotetext{
${ }^{1}$ Enfermeira. Mestre em Biociências e Saúde. Professora Assistente do Curso de Enfermagem. Membro do Grupo de Pesquisa em Saúde Materno-Infantil. E-mail: zanem2010@hotmail.com

${ }^{2}$ Enfermeira. Membro do Grupo de Pesquisa em Saúde Coletiva. E-mail: brunamazotti@hotmail.com

${ }^{3}$ Bióloga. Doutora em Ciências. Professora Adjunta do Curso de Enfermagem e do Programa de Pós-Graduação stricto sensu em Ensino e em Saúde Pública em Região de Fronteira. Membro do Grupo de Pesquisa em Saúde Coletiva. E-mail: aazilly@, hotmail.com

${ }^{4}$ Enfermeiro. Mestre em Ciências da Saúde. Professor Assistente do Curso de Enfermagem. Membro do Grupo de Pesquisa em Saúde Coletiva. E-mail: heelfer@gmail.com

${ }^{5}$ Enfermeiro. Doutor em Ciências. Professor Adjunto do Curso de Enfermagem e do Pragrama de Pós-Graduação Stricto Sensu em Saúde Pública em Região de Fronteira. Líder do Grupo de Pesquisa em Saúde Materno-Infantil. E-mail: calenf3@gmail.com
} 
approaches to studies on fetal health are relevant and important in terms of public health as an indicator of quality of prenatal care and childbirth assistance.

Keywords: Stillbirth. Birth. Risk factors.

\section{Introdução}

O óbito fetal é definido como a morte de um produto da concepção antes da expulsão ou da extração completa do corpo da mãe, com peso ao nascer igual ou superior a 500 gramas. Quando não houver informações sobre o peso ao nascer, deve-se considerar a idade gestacional de 22 semanas ou mais. Já em casos em que se desconhecem informações sobre o peso ao nascer e idade gestacional, considerar o comprimento corpóreo de 25 centímetros cabeçacalcanhar ou mais (BRASIL, 2009).

Estima-se que mais de quatro milhões de óbitos fetais ocorram anualmente no mundo, sendo a maioria em países em desenvolvimento e subdesenvolvidos. Contudo, embora se contabilize este alto número, verifica-se a redução da incidência de natimortos em pelo menos dez vezes nos últimos 50 anos (GOLDENBERG; MCCLURE; BELIZÁN, 2009; SAMPAIO; SOUZA, 2010).

Os avanços tecnológicos relacionados a métodos diagnósticos e as mudanças em protocolos assistenciais e de investigação prénatal tem propiciado a oferta do atendimento e seguimento à gestante de forma resolutiva e com qualidade, deste modo, compreende-se que este deveria reduzir complicações as quais desencadeiam a morte fetal (CABAR et al., 2008; FONSECA; COUTINHO, 2010). Nesse sentido, as melhorias globais na atenção primária possibilitam organizar as redes de atenção à saúde materna e infantil, considerando que a captação precoce da gestante e da criança são elementos essenciais para a atenção à saúde de qualidade (PARANÁ, 2012).

A morte fetal no Brasil é considerada um grave problema de saúde pública. Para tanto, as secretarias de saúde de diversos estados brasileiros têm buscado ampliar a assistência materna e infantil, baseada na estratégia ministerial da Rede Cegonha (BRASIL, 2011). O estado de São Paulo, por exemplo, criou a Rede Mãe Paulistana; o Rio Grande do Sul, a Rede Cegonha RS; o Rio de Janeiro, a Cegonha Carioca; o Paraná, a Rede Mãe Paranaense; entre outros. Este último programa foi implantado em 2012 com o objetivo de melhorar a assistência ao binômio mãe e filho, reduzir de forma acentuada as morbimortalidades, por meio do cuidado na gestação, parto e puerpério e seguimento às crianças menores de um ano (HUÇULAK; PETERLINI, 2014; PARANÁ, 2012).

A experiência de vários países tem demonstrado diferenças entre a mortalidade fetal e neonatal quanto às variáveis, escolaridade materna, raça, idade materna, condições de vida, entre outras. Sendo assim, aponta-se que a baixa escolaridade, a cor da pele materna preta ou parda, a adolescência ou a gestante em idade avançada e as precárias condições socioeconômicas perfazem indicativos de aumento dos índices de mortalidade infantil e fetal (PARANÁ, 2012).

Além desses fatores, as alterações fetais como o crescimento restrito intrauterino e malformações congênitas, e as morbidades maternas como as síndromes hipertensivas e hemorrágicas, aumentam significativamente a probabilidade de desfecho gestacional desfavorável, resultando em mortalidade fetal (KLEIN et al., 2012; YILDIRIM et al., 2014).

Considerando esse um grave problema de saúde pública em termos de indicador da qualidade da assistência pré-natal e ao parto, o presente estudo apresenta como objetivo analisar os óbitos fetais de municípios da nona regional de saúde do Paraná. 


\section{Materiais e Métodos}

Trata-se de uma pesquisa descritiva com abordagem quantitativa, realizada no primeiro semestre de 2015.

O cenário de estudo foi composto por municípios da região oeste do estado do Paraná, pertencentes a nona regional de saúde de Foz do Iguaçu, quais sejam: Foz do Iguaçu, Santa Terezinha de Itaipu, São Miguel do Iguaçu, Medianeira, Matelândia, Ramilândia, Serranópolis do Iguaçu, Itaipulândia e Missal.

Foz do Iguaçu é um município de grande porte com cerca de 260.000 habitantes. Possui uma população flutuante de aproximadamente 700.000 habitantes por pertencer a uma região de tríplice fronteira. Os municípios de Santa Terezinha de Itaipu, São Miguel do Iguaçu e Medianeira são considerados municípios de pequeno porte 2 , ou seja, a população de cada um soma entre 20.000 a 50.000 habitantes. Já os municípios de Ramilândia, Serranópolis do Iguaçu, Missal e Itaipulândia são considerados municípios de pequeno porte 1 (menos de 20.000 habitantes) (IBGE, 2010).

Os critérios de inclusão para a presente pesquisa foram todos os óbitos fetais ocorridos em municípios da nona regional de saúde no ano de 2010 a 2013. Foram excluídos os óbitos infantis e adultos (por todas as causas, como, externa, por problemas cardiovasculares, por câncer, entre outros) e os óbitos fetais em municípios não pertencentes a nona regional de saúde.

A coleta de dados foi realizada na base de dados do Departamento de Informática do Sistema Único de Saúde (DATASUS) pelo Sistema de Informação de Nascidos Vivos (SINASC), e Sistema de Informação sobre Mortalidade (SIM), além dos relatórios do Comitê de Mortalidade Materno e Infantil da nona Regional de Saúde.

Para a busca dos dados utilizou-se um instrumento estruturado, contendo as seguintes variáveis: município; número de nascidos vivos; número de óbitos fetais; sexo e peso do feto; duração da gestação; tipo de gravidez; tipo de parto; escolaridade e idade materna.
Os dados foram tabulados e analisados de forma descritiva para em seguida serem discutidos com base nas literaturas e de acordo com os protocolos do programa Rede Mãe Paranaense, programa vigente atualmente no estado do Paraná para a atenção à saúde materno-infantil (PARANÁ, 2012).

Salienta-se que, a presente pesquisa faz parte de um projeto maior, multicêntrico, intitulado "Análise do processo de implantação e desenvolvimento do Programa Rede Mãe Paranaense" o qual está financiado peloConselho NacionaldeDesenvolvimentoCientífico e Tecnológico - CNPq. Recebeu aprovação do Comitê de Ética e Pesquisa da Universidade Estadual do Oeste do Paraná, sob o parecer 544.107/2014, atendeu as normas da resolução 466/2012, a qual envolve pesquisas com seres humanos.

\section{Resultados e Discussão}

No período de 2010 a 2013 a nona regional de saúde do estado do Paraná contabilizou 194 óbitos fetais, chegando ao índice (médio) de 7,7 óbitos por 1000 Nascidos Vivos (NV). Neste mesmo período, o estado do Paraná, apresentou 8,9 óbitos fetais por $1000 \mathrm{NV}$, sendo que a regional de Jacarezinho (décima nona) teve o maior índice com 13,3 e a regional de Francisco Beltrão (oitava) o menor índice do estado com 6,5. O Brasil, entre 2010 a 2013, apresentou um índice de 11,0 óbitos fetais por $1000 \mathrm{NV}$.

Dentre os municípios pertencentes a nona regional de saúde do Paraná, antes da implantação do Programa Rede Mãe Paranaense, ou seja, 2010, Itaipulândia apresentou o maior índice de óbito fetal e os municípios de Ramilândia e Serranópolis do Iguaçu não apresentaram nenhum óbito fetal. Ressalta-se que todos os municípios da nona regional pactuaram com o referido programa.

Ao realizar uma avaliação após a implantação do programa, verificou-se que Serranópolis do Iguaçu e Missal não apresentaram nenhum óbito fetal, contudo, Itaipulândia apresentou novamente o maior índice de óbitos fetais com 25,6/1000 NV. 
Tabela 1 - Coeficiente de mortalidade fetal entre os municípios da nona regional de saúde do estado do Paraná no período de 2010 a 2013. Foz do Iguaçu, PR, 2015.

\begin{tabular}{l|cccccccccc}
\hline & $\begin{array}{c}\text { Foz do } \\
\text { Iguaçu }\end{array}$ & Itaipulândia & Matelândia & Medianeira & Missal & Ramilândia & STI & SMI & Serranópolis & $\begin{array}{c}\text { Regional } \\
\text { de Saúde }\end{array}$ \\
\hline $\mathbf{2 0 1 0}$ & 6,7 & 14,9 & 8,1 & 8,1 & 9,2 & 0 & 3,6 & 2,6 & 0 & 6,8 \\
$\mathbf{2 0 1 1}$ & 5,1 & 7,4 & 21,7 & 5,1 & 0 & 0 & 7,4 & 18,9 & 0 & 6,4 \\
$\mathbf{2 0 1 2}$ & 8,8 & 6,6 & 7,9 & 14,9 & 0 & 26,3 & 16,6 & 2,6 & 0 & 9,1 \\
$\mathbf{2 0 1 3}$ & 8,4 & 25,6 & 12,2 & 4,4 & 0 & 0 & 16,5 & 2,8 & 0 & 8,3 \\
\hline
\end{tabular}

Legenda: Santa Terezinha de Itaipu (STI); São Miguel do Iguaçu (SMI).

Fonte: DATASUS (2015).

Conforme a tabela 1, a comparação entre os índices dos municípios da nona regional com o índice geral da regional de saúde estudada, demonstra que os municípios de Foz do Iguaçu, Santa Terezinha de Itaipu, São Miguel do Iguaçu, Serranópolis do Iguaçu e Ramilândia, em 2010, apresentaram índices baixos em relação à média da regional. Em 2013, os municípios de Medianeira, Missal, Ramilândia, São Miguel do Iguaçu e Serranópolis do Iguaçu mostraram índices menores.

O programa Rede Mãe Paranaense foi instituído pela Secretaria de Estado do Paraná. Essa rede de atenção materno infantil é um dos compromissos assumidos no Plano de Governo para a Saúde entre 2011 a 2014. Esta rede de atenção refere-se a um conjunto de ações que envolvem a captação precoce da gestante; o acompanhamento prénatal, com no mínimo sete consultas; realização de exames laboratoriais e de imagem (urina I; urocultura; VDRL para detecção da sífilis; dosagem de hemoglobina; hematócrito; dosagem de glicose; pesquisa de antígeno de superfície do vírus de hepatite B - HBsAg; pesquisa de anti HBC IgG e IgM; pesquisa de anticorpo anti-HIV1 + HIV2 - ELISA; sorologia para toxoplasmose $\operatorname{IgG}$ e IgM; prova de avidez para IgG; ultrassonografia obstétrica; teste indireto de antiglobulina humana TIA; determinação da curva glicêmica; dosagem de proteínas - urina 24h; dosagem de ureia; dosagem de creatinina; dosagem de ácido úrico; desidrogenase láctica LDH; eletrocardiograma; contagem de plaquetas; ultrassonografia obstétrica com doppler; e tococardiografia anteparto) para apoio e diagnóstico; estratificação de risco das gestantes e das crianças; atendimento em ambulatório especializado para as gestantes e crianças de risco e garantia do parto por meio de um sistema de vinculação ao hospital conforme o risco gestacional (PARANÁ, 2012).

Considerando os desafios para lidar com a assistência voltada ao binômio mãe e filho, a implantação desse programa veio somar e organizar metas para melhor resolutividade assistencial nas diferentes esferas de atenção. $\mathrm{Na}$ esfera primária, o serviço deve ser o ordenador dos cuidados, com ações assistenciais de pré-natal e puerpério e acompanhamento do crescimento e desenvolvimento das crianças, em especial no seu primeiro ano de vida. Em nível secundário, realiza o acompanhamento das gestantes e crianças de risco em ambulatórios especializados com equipe multiprofissional. E ainda, em nível terciário, busca a ampliação de leitos de unidade de terapia intensiva adulto e neonatal, assim como, a vinculação das gestantes de risco em hospitais para a atenção às intercorrências e ao parto (PARANÁ, 2012).

Fonseca e Coutinho (2010) destacam a relevância de se conhecer os índices de mortalidade fetal de uma determinada população, pois este se apresenta como um importante indicador das condições socioeconômicas e qualidade da assistência pré- 
natal. Assim, aponta-se que a morte fetal pode estar associada a diversas variáveis socioeconômicas como a renda familiar, classe social da mãe e do pai, características maternas como idade e paridade, antecedentes obstétricos e perinatais.

A avaliação efetiva da qualidade de vida de uma determinada sociedade reporta-se a uma tendência temporal de queda da morbimortalidade. Experiências de vários países têm demonstrado que há diferenças entre a mortalidade quando se relaciona à escolaridade da mãe, às condições de vida e ao acesso aos serviços de saúde em tempo oportuno (PARANÁ, 2012).

A tabela 2 apresenta a incidência e características dos óbitos fetais em relação aos fatores do feto entre os municípios da nona regional de saúde, com exceção de Ramilândia que não apresentou óbitos fetais entre 2010 e 2013.

Tabela 2 - Caracterização dos óbitos fetais ocorridos entre 2010 e 2013 em municípios da nona regional de saúde relacionado com variáveis fetais. Foz do Iguaçu, PR, 2015.

\begin{tabular}{|c|c|c|c|c|c|c|c|c|c|c|c|c|c|c|c|c|c|c|c|c|c|c|c|c|c|c|c|c|c|c|c|c|}
\hline \multirow{3}{*}{ Variáveis } & \multicolumn{4}{|c|}{ Foz do Iguaçu } & \multicolumn{4}{|c|}{ Matelândia } & \multicolumn{4}{|c|}{ Medianeira } & \multicolumn{4}{|c|}{ STI } & \multicolumn{4}{|c|}{ Serranópolis } & \multicolumn{4}{|c|}{ Itaipulândia } & \multicolumn{4}{|c|}{ Missal } & \multicolumn{4}{|c|}{ SMI } \\
\hline & \multicolumn{2}{|c|}{2010} & \multicolumn{2}{|c|}{2013} & \multicolumn{2}{|c|}{2010} & \multicolumn{2}{|c|}{2013} & \multicolumn{2}{|c|}{2010} & \multicolumn{2}{|c|}{2013} & \multicolumn{2}{|c|}{2010} & \multicolumn{2}{|c|}{2013} & \multicolumn{2}{|c|}{2010} & \multicolumn{2}{|c|}{2013} & \multicolumn{2}{|c|}{2010} & \multicolumn{2}{|c|}{2013} & \multicolumn{2}{|c|}{2010} & \multicolumn{2}{|c|}{2013} & & 010 & & 013 \\
\hline & & $\%$ & $n$ & $\%$ & $\mathbf{n}$ & $\%$ & & $\%$ & & $\%$ & $\mathbf{n}$ & $\%$ & & $\%$ & & $\%$ & & $\%$ & & $\%$ & $\mathbf{n}$ & $\%$ & $\mathbf{n}$ & $\%$ & & $\%$ & & $\%$ & & $\%$ & & $\%$ \\
\hline OF & & & & & & & & & & & & & & & & & & & & & & & & & & & & & & & & \\
\hline Mascu & 18 & & 22 & 59 & 2 & 100 & 3 & 100 & 3 & 60 & 2 & 67 & 1 & 100 & 1 & 20 & 0 & 0 & & & 1 & 50 & 3 & 75 & 1 & 100 & & 0 & 0 & 0 & 1 & 50 \\
\hline $\mathrm{Fe}$ & 11 & 38 & 15 & 41 & 0 & 0 & 0 & 0 & 2 & 40 & 1 & 33 & ( & 0 & 4 & 80 & 1 & 100 & & 0 & 1 & 50 & 1 & 25 & ? & 0 & 0 & 0 & e & 100 & 1 & 50 \\
\hline Peso - gramas & & & & & & & & & & & & & & & & & & & & & & & & & & & & & & & & \\
\hline & 2 & & 1 & 3 & 0 & 0 & 0 & 0 & 1 & 20 & 1 & 33 & 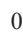 & 0 & 0 & 0 & 0 & 0 & & & 0 & 0 & 1 & 25 & & 0 & & & & 0 & 0 & 0 \\
\hline & 14 & & 16 & 43 & 1 & 5 & 2 & 67 & ( & 0 & 0 & c & & 100 & 1 & 20 & 1 & 100 & & 0 & 0 & & & 25 & & 0 & & 0 & & 100 & 1 & 50 \\
\hline & 4 & 14 & 12 & 32 & 1 & 50 & 1 & 33 & 1 & 20 & 2 & 67 & ( & 0 & 2 & 40 & 0 & 0 & 0 & 0 & 1 & 50 & & 25 & & 100 & 0 & 0 & & 0 & 0 & 0 \\
\hline & 6 & 21 & 8 & 22 & 0 & 0 & 0 & 0 & 3 & 60 & 0 & 0 & & 0 & 2 & 40 & 0 & ( & & 0 & 1 & 50 & 1 & 25 & 0 & 0 & 0 & 0 & & 0 & 1 & 50 \\
\hline & 3 & 10 & 0 & 0 & 0 & 0 & 0 & 0 & ( & 0 & 0 & 0 & & 0 & 0 & & 0 & & 0 & 0 & 0 & & 0 & & & 0 & 0 & 0 & & 0 & 0 & 0 \\
\hline & & & & & & & & & & & & & & & & & & & & & & & & & & & & & & & & \\
\hline$<22$ & 3 & 10 & 3 & 8 & 0 & 0 & 0 & 0 & ( & 0 & 0 & 0 & & 0 & 0 & 0 & 0 & 0 & 0 & 0 & 0 & 0 & 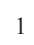 & 25 & & 0 & & 0 & & 100 & 0 & 0 \\
\hline 22 a 27 & 10 & 35 & 6 & 16 & 1 & 50 & 0 & 0 & 1 & 20 & 1 & 33 & ( & 0 & 0 & 0 & 0 & 0 & 0 & 0 & 0 & 0 & 1 & 25 & 1 & 100 & 0 & 0 & 0 & 0 & 0 & 0 \\
\hline & 12 & 41 & 22 & 59 & 1 & 50 & 2 & 67 & 2 & 40 & 2 & 67 & 1 & 100 & 3 & 60 & 1 & 100 & 0 & 0 & 1 & 50 & 0 & 0 & 0 & 0 & 0 & 0 & & 0 & 2 & 100 \\
\hline 37 a 41 & 3 & 10 & 4 & 11 & 0 & 0 & 1 & 33 & 2 & 40 & 0 & 0 & ( & 0 & 2 & 40 & 0 & 0 & 0 & 0 & 1 & 50 & 2 & 50 & 0 & 0 & 0 & 0 & 0 & 0 & 0 & 0 \\
\hline & 1 & 4 & 1 & 3 & 0 & 0 & 0 & 0 & ( & 0 & 0 & 0 & ( & 0 & 0 & 0 & 0 & 0 & & 0 & 0 & 0 & 0 & 0 & 0 & 0 & 0 & 0 & & 0 & 0 & 0 \\
\hline & 0 & 0 & 1 & 3 & 0 & 0 & 0 & 0 & 0 & 0 & 0 & 0 & 0 & 0 & 0 & 0 & 0 & 0 & 0 & 0 & 0 & 0 & 0 & 0 & 0 & 0 & 0 & 0 & 0 & 0 & 0 & 0 \\
\hline Total & 29 & 100 & 37 & 100 & 2 & 100 & 3 & 100 & & 100 & 3 & 100 & & 100 & 5 & 100 & 1 & 100 & 0 & 0 & 2 & 100 & 4 & 100 & & 100 & 0 & 0 & & 100 & 2 & 100 \\
\hline
\end{tabular}

Legenda: Santa Terezinha de Itaipu (STI); São Miguel do Iguaçu (SMI); Idade Gestacional (IG); Óbito Fetal (OF). Fonte: DATASUS (2015) e Paraná (2015).

Conforme apresenta a tabela 2, o município de Foz do Iguaçu, sede da nona regional de saúde apresentou o maior número de óbitos fetais, porém este apresenta o maior número de nascimentos. O referido município pertence a uma região de tríplice fronteira, ou seja, uma região vulnerável por manter-se aberta a inúmeros indivíduos podendo levar a desassistência da população permanente pela morosidade dos serviços de saúde e falta de resolutividade ao atender uma população muito maior e não prevista pelo sistema de saúde (ALBUQUERQUE, 2012).
Entre os municípios estudados constatou-se que cinco obtiveram aumento no número de óbitos fetais, sendo que, Santa Terezinha de Itaipu foi o que demonstrou maior relevância ao apresentar um aumento de cinco vezes mais.

Quanto ao sexo do feto que teve como desfecho o óbito, verificou-se a predominância do sexo masculino, com peso fetal ente 500 a 1499 gramas e IG de 28 a 36 semanas. Segundo ChiavegattoFilho e Laurenti (2012), os resultados encontrados estão de acordo com as pesquisas sobre óbitos fetais 
masculinos, em que as categorias relacionadas com maior risco de óbitos fetais masculinos foram idade materna entre 10 e 14 anos, nenhuma escolaridade e gestação com menos de 22 semanas.

Embora se observe um número superior de óbitos fetais com baixo peso e prematuros, houve 22 óbitos de fetos com peso acima de 2499 gramas, ressaltando o agravante na atenção à saúde da gestante, a qual desencadeia a morbimortalidade fetal em condições evitáveis.

Camargo (2008) em seu estudo ressalta que aproximadamente $86 \%$ dos óbitos fetais ocorridos em 2006 em São Paulo tinham peso entre 500 e 900 gramas, seguidos pelas faixas de 1000 a 1499 gramas e de 1500 a 1999 gramas. Entretanto, chamase atenção nesta pesquisa o fato que, um quarto dos fetos que evoluíram a óbito não tinha baixo peso (abaixo de 2500 gramas), e ainda, que 13\% tinham mais de 3000 gramas.
Outro estudo mostrou que o risco de óbito fetal aumenta em 5,72 vezes quando o peso do feto ou do recém-nascido é inferior a 2500 gramas (OLIVEIRA; GAMA; SILVA, 2010).

Em Foz do Iguaçu, foram identificados três casos de óbitos fetais com IG maior de 42 semanas. As gestações prolongadas ocorrem em aproximadamente $10 \%$ das gestações e podem causar acometimentos irreversíveis para a mãe e para o feto, aumentando a morbimortalidade de ambos. A relação da gestação prolongada e morte fetal, muitas vezes, ocorrem por insuficiência placentária ou ainda a aspiração de mecônio (MATTHES, 2010).

A tabela 3 apresenta a incidência e características dos óbitos fetais em relação aos fatores maternos dos municípios da nona regional de saúde, com exceção Ramilândia que não apresentou óbitos fetais em 2010 e 2013.

Tabela 3 - Incidência e caracterização dos óbitos fetais ocorridos entre 2010 e 2013 em municípios da nona regional de saúde do estado do Paraná relacionados a variáveis maternas. Foz do Iguaçu, PR, 2015

\begin{tabular}{|c|c|c|c|c|c|c|c|c|c|c|c|c|c|c|c|c|c|c|c|c|c|c|c|c|c|c|c|c|c|c|c|c|}
\hline \multirow{4}{*}{$\begin{array}{l}\text { Variáveis } \\
\text { IM - anos }\end{array}$} & \multicolumn{4}{|c|}{ Foz do Iguaçu } & \multicolumn{4}{|c|}{ Matelândia } & \multicolumn{4}{|c|}{ Medianeira } & \multicolumn{4}{|c|}{ STI } & \multicolumn{4}{|c|}{ Serranópolis } & \multicolumn{4}{|c|}{ Itaipulândia } & \multicolumn{4}{|c|}{ Missal } & \multicolumn{4}{|c|}{ SMI } \\
\hline & \multicolumn{2}{|c|}{2010} & \multicolumn{2}{|c|}{2013} & \multicolumn{2}{|c|}{2010} & \multicolumn{2}{|c|}{2013} & \multicolumn{2}{|c|}{2010} & \multicolumn{2}{|c|}{2013} & \multicolumn{2}{|c|}{2010} & \multicolumn{2}{|c|}{2013} & \multicolumn{2}{|c|}{2010} & \multicolumn{2}{|c|}{2013} & \multicolumn{2}{|c|}{2010} & \multicolumn{2}{|c|}{2013} & \multicolumn{2}{|c|}{2010} & 20 & & & 10 & & 13 \\
\hline & & $\%$ & & $\%$ & $\mathbf{n}$ & $\%$ & $\mathbf{n}$ & $\%$ & $\mathbf{n}$ & $\%$ & n & $\%$ & & $\%$ & & $\%$ & & $\%$ & & & & $\%$ & & $\%$ & & $\%$ & & & & & & $\%$ \\
\hline & & & & & & & & & & & & & & & & & & & & & & & & & & & & & & & & \\
\hline 20 a 39 & 27 & 93 & 25 & 68 & 2 & 100 & 3 & 100 & 5 & 100 & 0 & 0 & 0 & 0 & 2 & 40 & 1 & 100 & 0 & 0 & 2 & 100 & 3 & 75 & 1 & 100 & 0 & 0 & 1 & 100 & 1 & 50 \\
\hline$>40$ & 0 & 0 & 2 & 5 & 0 & 0 & 0 & 0 & 0 & 0 & 2 & 67 & 0 & 0 & 3 & 60 & 0 & 0 & 0 & 0 & 0 & 0 & 0 & 0 & 0 & 0 & 0 & 0 & 0 & 0 & 0 & 0 \\
\hline Ignorada & 0 & 0 & 0 & 0 & 0 & 0 & 0 & 0 & 0 & 0 & 0 & 0 & 1 & 100 & 0 & 0 & 0 & 0 & 0 & 0 & 0 & 0 & 0 & 0 & 0 & 0 & 0 & 0 & 0 & 0 & 1 & 50 \\
\hline EM - anos & & & & & & & & & & & & & & & & & & & & & & & & & & & & & & & & \\
\hline 4 a 7 & 9 & 31 & 7 & 19 & 0 & 0 & 0 & 0 & 1 & 20 & 0 & 0 & 0 & 0 & 0 & 0 & 0 & 0 & 0 & 0 & 2 & 100 & 0 & 0 & 0 & 0 & 0 & 0 & 0 & 0 & 1 & 50 \\
\hline 8 a 11 & 8 & 28 & 20 & 54 & 1 & 50 & 3 & 100 & 2 & 40 & 2 & 67 & 0 & 0 & 1 & 20 & 0 & 0 & 0 & 0 & 0 & 0 & 3 & 75 & 0 & 0 & 0 & 0 & 0 & 0 & 0 & 0 \\
\hline$>12$ & 0 & 0 & 5 & 14 & 0 & 0 & 0 & 0 & 2 & 40 & 1 & 33 & 0 & 0 & 3 & 60 & 0 & 0 & 0 & 0 & 0 & 0 & 0 & 0 & 1 & 100 & 0 & 0 & 0 & 0 & 1 & 50 \\
\hline Ignorado & 0 & 0 & 0 & 0 & 1 & 50 & 0 & 0 & 0 & 0 & 0 & 0 & 1 & 100 & 1 & 20 & 0 & 0 & 0 & 0 & 0 & 0 & 0 & 0 & 0 & 0 & 0 & 0 & 0 & 0 & 0 & 0 \\
\hline Gravidez & & & & & & & & & & & & & & & & & & & & & & & & & & & & & & & & \\
\hline & 25 & 86 & 35 & 95 & 2 & 100 & 3 & 100 & 5 & 100 & 3 & 100 & 1 & 100 & 5 & 100 & 1 & 100 & 0 & 0 & 2 & 100 & 4 & 100 & 1 & 100 & 0 & 0 & 1 & 100 & 2 & 100 \\
\hline Dupla & 4 & 14 & 2 & 5 & 0 & 0 & 0 & 0 & 0 & 0 & 0 & 0 & 0 & 0 & 0 & 0 & 0 & 0 & 0 & 0 & 0 & 0 & 0 & 0 & 0 & 0 & 0 & 0 & 0 & 0 & 0 & 0 \\
\hline Parto & & & & & & & & & & & & & & & & & & & & & & & & & & & & & & & & \\
\hline Cesárea & 7 & 24 & 10 & 27 & 1 & 50 & 2 & 67 & 3 & 60 & 1 & 33 & 1 & 100 & 4 & 80 & 1 & 100 & 0 & 0 & 2 & 100 & 0 & 0 & 0 & 0 & 0 & 0 & 0 & 0 & 1 & 50 \\
\hline Total & 29 & 100 & 37 & 100 & 2 & 100 & 3 & 100 & 5 & 100 & 3 & 100 & 1 & 100 & 5 & 100 & 1 & 100 & $\mathbf{0}$ & $\mathbf{0}$ & 2 & 100 & 4 & 100 & 1 & 100 & 0 & 0 & 1 & 100 & 2 & 100 \\
\hline
\end{tabular}

Legenda: Santa Terezinha de Itaipu (STI); São Miguel do Iguaçu (SMI); Escolaridade Materna (EM); Idade Materna (IM).

Fonte: DATASUS (2015) e Paraná (2015)..

Em relação aos dados maternos encontrados nestes municípios, verificou-se a predominância da idade da mãe entre 20 a 39 anos, porém em 2013, Medianeira e Santa Terezinha de Itaipu identificaram óbitos fetais entre mães com idade acima de 40 anos.

A idade materna é uma informação importante para avaliação dos riscos de ocorrência das perdas 
fetais, a exemplo do que se observa na mortalidade infantil, em que as relações já são mais conhecidas, sendo que os maiores riscos ocorrem para mães muito jovens e aquelas com idades mais avançadas (CAMARGO, 2008). Embora para os óbitos fetais esse conhecimento ainda é restrito, a gravidez na adolescência (entre 10 e 19 anos) e em mulheres com mais de 35 anos pode ser considerada um fator de risco para sérias complicações materna e fetal (SALOOJEE; COOVADIA, 2015).

Andrade et al. (2009) destaca uma associação significante entre óbito fetal e idade materna, identificando uma chance de 2,5 vezes maior dos natimortos serem filhos de mulheres com 35 anos ou mais. Em relação ao nível de escolaridade da mãe, foi apontada uma chance de 2,3 vezes maior dos natimortos serem filhos de mulheres com escolaridade menor que oito anos.

Trindade et al. (2011) analisou os óbitos fetais entre os anos de 2000 e 2007 no município de Pato Branco, e a maioria dos casos abrangeu a faixa etária materna de 31 a 40 anos. Nesta faixa etária observa-se a queda das taxas de fecundidade, bem como o aumento da ocorrência de gestações de alto risco.

$\mathrm{Na}$ regional de saúde desta pesquisa, em 2010, pode-se mostrar maior frequência de baixa escolaridade materna, pois para a maioria dos óbitos fetais as mães tinham menos de três anos de estudo. Em 2013 este panorama se modificou, pois, a maioria dos óbitos ocorreu com mães com escolaridade entre oito a onze anos. A secretaria estadual de saúde do Paraná apresentou dados estatísticos demonstrando que existe um risco relativo de morte de 2,5 vezes maior para filhos com mães de baixa escolaridade (PARANÁ, 2012).

Outro fator analisado foi em relação ao tipo de gestação, constatando gestação única para o maior número de óbitos em todos os municípios no período estudado. Já quanto ao tipo de parto, os municípios de Medianeira, Serranópolis do Iguaçu, Santa Terezinha de Itaipu, Itaipulândia e Matelândia realizaram para a maioria das mulheres o parto cirúrgico.

Sabe-se que o parto cirúrgico não deve ser recomendado para a maior parte das gestantes. Contudo, diante de agravos maternos os quais põe em risco a vida de ambos - mãe e feto - recomenda-se a resolução do nascimento o mais precoce possível. Se este for o parto cirúrgico, o mesmo deverá ser realizado em um centro de atendimento estruturado e com recursos humanos adequados para atender complexidades (SAMPAIO; SOUZA, 2010).

Em um estudo realizado com 258 gestantes com óbitos fetais, verificou que $71(27,5 \%)$ foram submetidas à cesariana. Dentre as indicações dessa via de parto, o Descolamento Prematuro da Placenta (DPP) prevaleceu com altos índices (49,3\%), seguido de síndromes hipertensivas $(14,1 \%)$, duas ou mais cesarianas anteriores $(14,1 \%)$, gestação múltipla (11,3\%), falhas de indução do parto $(9,9 \%)$ e outras indicações de menor frequência. (SAMPAIO; SOUZA, 2010). Ressalta-se que, os partos cirúrgicos devem apenas ser realizados em circunstâncias bem definidas pela equipe médica e assistencial, pois cesarianas anteriores favorecem significativamente o risco para o óbito fetal em gestações futuras, (BLACK; SHETTY; BHATTACHARYA, 2008; MORAITIS et al., 2015; WOOD et al., 2008) podendo aumentar em 23\% (O’NEILL et al., 2013).

No estado do Paraná, após uma análise dos nascimentos e da mortalidade materna e infantil no período de 2006 a 2010, identificou-se a necessidade de estabelecer a estratificação de risco de forma diferenciada para gestantes e crianças. Assim, foram definidos três graus de risco, quais sejam: Risco Habitual, Risco Intermediário e Alto Risco (PARANÁ, 2012).

O risco habitual envolve as gestantes e crianças que não apresentam fatores de risco individual, de doença ou agravo, de história reprodutiva e sociodemográficos. O risco intermediário referese ao grupo que apresenta, ou, que seja filho de 
mães com fatores relacionados à idade menor que 20 ou maior que 40 anos, raça negra ou indígena, baixa escolaridade e história reprodutiva anterior de multiparidade ou filhos mortos. Ressalta-se que, este grupo de gestantes apresentam maiores chances de desenvolverem morbidades, como síndromes hipertensivas (em relação à idade) e a anemia falciforme para as afrodescendentes, todas quais, podem levar ao sofrimento fetal e consequentemente a morte (PARANÁ, 2012).

E o grupo considerado de alto risco abrange uma condição clínica materna pré-existente (cardiopatias, pneumopatia, entre outras), intercorrências obstétricas atuais (síndrome hipertensivas, placenta prévia, trabalho de parto prematuro, isoimunização $\mathrm{Rh}$, entre outros) e de nascimento (malformação congênita, prematuridade, asfixia grave, entre outros) (PARANÁ, 2012).

Através da estratificação de risco se define a sua vinculação ao pré-natal e ao hospital para o atendimento de intercorrências na gestação, no momento do parto e a criança até o primeiro ano de vida. A caracterização de uma situação de risco não implica necessariamente em referenciar a gestante para acompanhamento em pré-natal de risco, mas devem ser reforçados os cuidados à saúde e a atenção da equipe interdisciplinar de saúde nos diferentes níveis de atenção (PARANÁ, 2012).

Alves et al. (2013) acrescentam que, pelo reconhecimento do risco da gestante e da criança, pode-se ampliar o olhar crítico e singularizado acerca das características de cada indivíduo, dentre elas as condições socioeconômicas e de saúde. Assim, será possível identificar suas principais necessidades e trabalhar junto a elas na promoção e proteção da saúde materna e fetal.

Na tentativa de minimizar a incidência de óbitos fetais, assim como, da mortalidade infantil, Trindade et al. (2011) indicam que a melhoria do controle das doenças no período gestacional e o melhor acompanhamento pré-natal poderiam ser medidas promotoras de evitabilidade, somado a completude de informações nas declarações de óbito como recursos para investigação e implementação de estratégias para o cuidado.

Os resultados deste estudo retratam que, para reduzir o número de óbitos fetais há necessidade de esforços concentrados, principalmente os relacionados na melhoria da assistência pré-natal e da assistência ao parto. Esses esforços incluem a implementação de medidas básicas de atenção à saúde, como assistência à gestante com qualidade, estruturação dos centros de atendimento as gestantes e parturientes, capacitação profissional, humanização no atendimento, qualificação da assistência ao recém-nascido em sala de parto, ampliação de unidades de terapia intensiva neonatal e manejo adequado do recém-nascido de risco (ANDRADE et al., 2009).

E ainda, a melhoria nos serviços de atenção primária, compreendendo-os como organizadores das Redes de Atenção à Saúde, neste contexto a Rede Mãe Paranaense, seria essencial para nortear a atenção à saúde com qualidade, para assim, o segmento materno e infantil receber a atenção à saúde de forma resolutiva e em tempo oportuno (PARANÁ, 2012).

\section{Conclusão}

A nona regional de saúde do Paraná demonstrou fragilidades na atenção à saúde da gestante durante o pré-natal, considerando o aumento da mortalidade fetal. Dos nove municípios, cinco tiveram aumento no número de óbitos fetais. Itaipulândia apresentou os piores índices para o período estudado e Santa Terezinha de Itaipu um aumento de cinco vezes entre 2010 e 2013.

Com a implantação do programa Rede Mãe Paranaense, espera-se que haja melhorias na estratificação do risco gestacional, na captação precoce da gestante, nos exames laboratoriais e de imagem e na capacitação profissional, refletindo consequentemente em melhores resultados para a saúde materna e fetal.

A construção de estudos sobre a saúde fetal e perinatal torna-se extremamente relevante, pois 
pesquisas como essas têm sido pouco exploradas, apesar de sua incontestável importância em termos de saúde pública.

Como fator limitador do estudo, aponta-se a precocidade desta avaliação da mortalidade fetal nesta regional de saúde, considerando o tempo desde a implantação e estruturação do Programa Rede Mãe Paranaense. Ao mesmo tempo, estudos como este poderão subsidiar pesquisas vindouras sobre a temática, possibilitando um maior panorama deste indicador quando relacionado às ações em saúde desenvolvidas a partir do referido programa.

\section{Referências}

ALBUQUERQUE, J. L. Limites e paradoxos da cidadania no território fronteiriço: o atendimento dos brasiguaios no sistema público de saúde em Foz do Iguaçu (Brasil). Geopolítica(s), Natal, v. 3, n. 2, p. $185-205,2012$.

ALVES, C. N.; RESEL, L. B.; SANFELICE, C.; BISOGNIN, P.; WILHELM, L. A.; ZANINI, R. R.; Perfil de gestantes assistidas no pré-natal de enfermagem em uma unidade básica de saúde. Revista de Pesquisa: Cuidado é Fundamental, Rio de Janeiro, v. 5, n. 3, p. 132-141, 2013.

ANDRADE, L. G.; AMORIM, M. M. R.; CUNHA, A. S. C; LEITE, S. R. F.; VITA, A. S. Fatores associados à natimortalidade em uma maternidade escola em Pernambuco: estudo caso-controle. Revista Brasileira de Ginecologia e Obstetrícia, Rio de Janeiro, v. 31, n. 6, p. 285-292, 2009.

BLACK, M.; SHETTY, A.; BHATTACHARYA, S. Obstetric outcomes subsequent to intrauterine death in the first pregnancy. An International Journal of Obstetrics and Gynaecology, Oxford, v. 115, n. 2, p. 269-274, 2008.

BRASIL. Ministério da Saúde. Manual dos comitês de prevenção do óbito infantil e fetal. 2. ed. Brasília, 2009.

BRASIL. Ministério da Saúde. Portaria $n^{\circ} 1.459$ de 24 de junho de 2011. Institui a Rede Cegonha. Brasília, 2011.
CABAR, F. R.; NOMURA, R. M. Y.; MACHADO, T. R. S.; ZUGAIB, M. Óbito fetal no descolamento prematuro da placenta: comparação entre dois períodos. Revista da Associação Médica Brasileira, São Paulo, v. 54, n. 3, p. 256-260, 2008.

CAMARGO, M. B. A. A natimortalidade e a mortalidade perinatal em São Paulo. São Paulo em Perspectiva. São Paulo, v. 22, n. 1, p. 30-47, jan./jun. 2008.

CHIAVEGATTO-FILHO, A. D. P.; LAURENTI, R. O sexo masculino vulnerável: razão de masculinidade entre os óbitos fetais brasileiros. Caderno de Saúde Pública, São Paulo, v. 28, n. 4, p. 720-728, 2012.

DATASUS. Departamento de informática do SUS. Paraná. Disponível em: <http://datasus. saude.gov.br/nucleos-regionais/parana $>$. Acesso em: 13 maio 2015.

FONSECA, S. C.; COUTINHO, F. S. E. Fatores de risco para mortalidade fetal em uma maternidade do Sistema Único de Saúde, Rio de Janeiro, Brasil: estudo caso-controle. Caderno de Saúde Pública, Rio de Janeiro, v. 26, n. 2, p. 240252, 2010.

GOLDENBERG, R. L.; MCCLURE, E. M.; BELIZÁN, J. M. Commentary: reducing the world's stillbirths. BMC Pregnancy and Childbirth, London, v. 9, s.1, 2009.

HUÇULAK, M. C.; PETERLINI, O. L. Rede mãe paranaense: relato de experiência. Revista Espaço para a Saúde, Londrina, v. 15, n. 1, p. 77-86, abr. 2014.

IBGE. 2010. Disponível em: <http://www.ibge. gov.br/home/estatistica/populacao/censo2010/ tabelas_pdf/total_populacao_parana.pdf $>$ Acesso em: 13 de maio 2015.

KLEIN, J. C.; MADI, M. J.; ARAÚJO, F. B.; ZATTI, H.; DAL BOSCO, S. D.; HENKE, N. C. Fatores de risco relacionados à mortalidade fetal. Revista da AMRIGS, Porto Alegre, v. 56, n. 1, p. 11-16, jan./mar. 2012. 
MATTHES, A. C. S. Gravidez prolongada: subsídios da literatura médica para uma defesa. Femina, Ribeirão Preto, v. 38, n. 8, p. 393-400, ago. 2010.

MORAITIS, A. A.; OLIVER-WILLIAMS, C.; WOOD, A. M.; FLEMING, M.; PELL, J. P.; SMITH, G. Previous caesarean delivery and the risk of unexplained stillbirth: retrospective cohort study and meta-analysis. An International Journal of Obstetrics and Gynaecology, Oxford, May, 2015.

OLIVEIRA, V. F. E; GAMA, N. G. S.; SILVA, P. F. M. C. Gravidez na adolescência e outros fatores de risco para mortalidade fetal e infantil no Município do Rio de Janeiro, Brasil. Caderno de Saúde Pública. Rio de Janeiro, v. 26, n. 3, p. 567-578, 2010.

O'NEILL, S. M.; KEARNEY, P. M.; KENNY, L. C.; KHASHAN, A. S.; HENRIKSEN, T. B.; LUTOMSKI, J. E.; GREENE, R. A. Caesarean delivery and subsequent stillbirth or miscarriage: systematic review and meta-analysis. PLoS One, San Francisco, v. 8, n. 1, e54588, 2013.

PARANÁ. Secretaria da Saúde. Regionais SESA $9^{a}$ $R S$ : Foz do Iguaçu. Disponível em: <http://www. saude.pr.gov.br/modules/conteudo/conteudo. php? conteudo $=2761>$. Acesso em: 13 maio 2015 .

PARANÁ. Secretaria de Estado da Saúde do Paraná. Linha guia Rede Mãe Paranaense: SESA (PR). Curitiba, 2012.

SALOOJEE, H.; COOVADIA, H. Maternal age matters: for a lifetime, or longer. The Lancet, London, v. 3, n. 7, p. 342-43, 2015.

SAMPAIO, A. G.; SOUZA, A. S. Indicação de cesarianas em óbito fetal. Revista Brasileira de Ginecologia e Obstetrícia, Recife, v. 32, n. 4, p. 169-175, 2010.

TRINDADE, L. L.; AMESTOY, S. C.; PICOLO, D.; FALCHETTI, G.; MILBRATH, M. V. Fatores de risco para morte fetal no município de Pato Branco (Brasil). Investigação e Educação em Enfermagem, Pato Branco, v. 29, n. 3, p. 441-458, 2011.
WOOD, S. L.; CHEN, S.; ROSS, S.; SAUVE, R. The risk of unexplained antepartum stillbirth in second pregnancies following caesarean section in the first pregnancy. An International Journal of Obstetrics and Gynaecology, Oxford, v. 115, n. 6, p. 726-731, May, 2008.

YILDIRIM, G.; ASICIOGLU, O.; GUNGORDUK, K.; TURAN, I.; ACAR, D.; ASLAN, H.; GUNAY, T. Subsequent obstetrics outcomes after intrauterine death during the first pregnancy. Journal of Maternal and Fetal Neonatal Medicine, London, v. 27, n. 10, p. 1029-1032, Jul. 2014. 